and labour tests, might make good Foresters under proper command, and they would be fully as available a nucleus for an army, in case the most unlikely of contingencies should occur, as any force which Canada at present possesses.

In 1963 and 1964 I visited forestry officials and forestry operations in Sweden, Finland and Soviet Russia. I decided, crudely and bumptiously, that not much of what Russia was doing was pertinent to home because their decision-making methods, transportation system and their large pool of forest labor had taken them into silvicultural and harvesting practices with little utility for Canada.

As for the Swedes, they were so damnably rational. Their labor relations were quite unlike ours. Their great concern for foreign trade was as keen as their concern for the environment. Their common sense was so evident in the thoroughness with which they staged public discussion and policy development in order to bring along the enlightened elements of the country on forestry issues, whether to do with forestry as involving sites or mills or sales. I saw their level of sophistication and inherent national unity as several decades ahead of ours.

Finland seemed more a place of kindred spirit to Canada. As a culmination of field trips and talks around the country. I spent a few hours with a Dr. Lahti, then the head of the government forest service. Naturally, I was contrasting the keener nurture and the much greater significance which the Finns gave to forestry than we did. Dr. Lahti was both sweet and precise. His reply went like this.

It was not always so. He had visited Canada several times ... and he mentioned men he'd met and forests and logging chances he had seen.
"Let me tell you," he said, "what I think about Canada. It will happen, as here. You will get real forestry when you are desperate for wood to harvest and I don't just mean private sector desperation or wood-farmers desperation but national desperation. Finland had two terrible squeezes: the first, the lighter one, was after we won our freedom and had to resurrect an economy torn by civil war. Out of it came policies which bettered opportunities for thousands of small bush farmers - part subsistence-farming, part logging and pulp cutting; we had a lot of sawmilling for export. The creation got underway of pulp and paper manufacturing on a fairly large scale. So we go into more and more real forestry exploitation, but it wasn't really tough and effective forest management. That wasn't to come until the worst squeeze; after losing the Karelian lands in ' 45 and having to resettle the people from there, and having to pay back the big reparations demanded. We had to get out of debt to the USSR.

What were we to base those repayment efforts on? We had small choices other than the forest industries. When we turned to the forests we realized that both our settlement policies and the neglect of the past, plus resettling the Karelians, had put us in a desperate situation. We didn't have that much wood or a good system of roads or a real grip on regeneration. We were desperate for wood; the whole nation knew it; and to the forester came to be heard, we became a power in the land. You will get forestry in Canada when your people as a whole realize, probably rather suddenly, that you're getting desperately short of useable forest. I think myself, said Dr. Lahti, you are 20 years or so away from that. . but remember, you'll get there!" Are we there? Is that your prime Lobbyist message?

\title{
New Active Members
}

L. Archambault, Environment Canada, C.P. 3800 , Ste. Foy, P.Q. G1V 2 Z2

D. A. Baardsen, Box 133, Hampton, N.B. EOG 1 Z0

R. B. Dettle, Prov. Forest Nursery, R.R. $\neq 6$, Fredericton, N.B. E3B $4 \times 5$

M. Boudoux, 1996 Chemin Demers, Bernieres, G0S 1 C0

G. Chantel, Centre de Res. For. Des Laurentides, P.Q. Box 3800, Ste. Foy, P.Q. G1V 4C7

A. H. Denton, Victoria Wood Industries, Box 1588, Grand Falls, N.B. EOJ $1 \mathrm{MO}$

R. G. Grover, Alberta Environment, Land Reclamation, 11th F1. Oxbridge Place, Edmonton, Alta T5K 2J6

J. B. Harrington, Petawawa National Forestry Inst., Chalk River, Ont. KOJ $1 \mathrm{JO}$

R. J. Jordon, Forsite Limited, 600 Main St., Yarmouth, N.S. B5A $1 \mathrm{~J} 7$

G. Laflamme, 1080 Route du Vallon, Box 3800 , Ste. Foy, P.Q. G1V $4 \mathrm{C} 7$

R. M. Marshall, Canadian Forest Products Ltd., 14th FI., 505 Burrard St., Vancouver, B.C. V7X $1 \mathrm{M} 4$

D. F. O'Brien, Forest Ext. Service, R.R. $\$ 5$, Fredericton, N.B. E3B $3 \mathrm{C} 7$

D. Ouellet, Centre de Res. For. Des Laurentides, CP 3800, Ste. Foy, P.Q. G1V 4C7

B. D. Prebble, 11 Mayo St., Grand Falls, Nfld. A2A 1L4

M. R. Roberge, Environment Canada, Box 3800, Ste. Foy, P.Q. G1V $2 Z 2$

A.D. Russell, Box 104, Pine Falls, Man. ROE 1 MO

E. D. Stinson, Box 43, Site 27, R.R. $=66$, Fredericton, N.B. E3B $4 \times 7$

J. Trencia, Centre de Res. For. des Laurentides, C.P. 3800, St., Foy P.Q. G1V 4 C7

H. S. Whitney, 1925 Casa Marcia, Victoria, B.C. V8N $2 \times 4$

G. C. Woollacott, Northwood Pulp \& Timber Ltd., Box 9000, Prince George, B.C. V2L 4W2

\section{New Student Members}

R. H. Ballance, 718 Beaverbrook St., Fredericton, N.B. E3B 1 Y2

\section{Woodlot Service (1978) Ltd.}

"All Matters Pertaining to Forestry"

GORDON B. YOUNG, B.Sc.F., M.F.

Registered Professional Forester

320 Maple Street

Bus.: 506 - 472-3396

Fredericton, N.B.

Home: 506 - $472-7721$ 


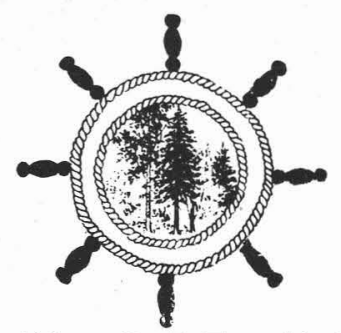

1981 Annual Meeting

CANADIAN INSTITUTE OF FORESTRY

September 26 - October 1, 1981

Hotel Nova Scotian, Halifax, N.S.

"Set Sail For Halifax"

\section{Saturday, September 26}

1:30 - 5.00 p.m. ClF Board of Directors Meeting

\section{Sunday, September 27}

9:00 - 5.00 p.m. CIF Board of Directors Meeting

2:00 - 7.00 p.m. Registration

8:00 - 10.00 p.m. Icebreaker

\section{Monday, September 28}

8:00 - 12.00 noon Registration

8:00 - 9.30 a.m. Buffet Breakfast

9:30 - 10.30 a.m. Opening Ceremonies

10:45 - 12.00 noon Business Meeting, Part I

$12: 00$ - 2.00 p.m. Luncheon

2:00 - 5.00 p.m. Keynote Addresses

— John Weslien, Chief

Forester

Billeruds AB, Sweden

-W. Lester Brown, Director of Woodlands Bowater Inc.

- Gordon Minnes Canadian Pulp and Paper Association

- J. Gordon Ogden III, Biology Professor Dalhousie University

\section{Tuesday, September 29}

9:00 - 12.00 noon Panel: "Intensive Forestry in Nova Scotia"

$12: 00$ - 2.00 p.m. Province of N.S. Luncheon

2:00 - 5.00 p.m. Panel: "Developments Across Canada

\section{Wednesday, September 30}

8:30 - 5.00 p.m. Field Tours. Choice of:

Tour\#1 - Harvesting and Forest Management on Crown Lands and Large Freehold

Tour\#2 -Forest Management on Small Woodlots, Christmas tree stands

Tour\#3 - Small wood-using industries

6:00 - 1.00 a.m. Lobster Dinner and Dance

Thursday, October 1

9:00 - 12.00 noon Working Group Meetings

12:00 - 2.00 p.m. Alumni Luncheons

2:00 - 4.30 p.m. Business Meeting, Part 2

6:30 - 7.30 p.m. Social Hour

7:30 - 9.00 p.m. Banquet

9:00 - 1.00 a.m. Dance

Friday, October 2 and Saturday, October 3 Possible Post-Conference Tour to Cape Breton

\section{Sunday, September 27}

11:30 - 2.30 p.m. Reception for Directors' Wives

2:00 - 7.00 p.m. Registration

8:00 - 10.00 p.m. Icebreaker

\section{Monday, September 28}

8:00 - 12.00 noon Registration

8:00 - 9.30 a.m. Buffet Breakfast

10:30 - 5.30 p.m. Tour of Annapolis Valley

\section{Tuesday, September 29}

10:00 - 11.30 a.m. Historic Walk, Craft demonstration

12:00 - 2.00 p.m. Province of Nova Scotia Luncheon

2:30 - 3.30 p.m. Fashion Show

\section{SPOUSES' PROGRAM}

\section{Wednesday, September 30}

10:00 - 12.00 noon Short Tours (City and Museums)

12:00 - 2.00 p.m. Lunch

2:00 - 4.00 p.m. Halifax Water Tour

6:00 - 1.00 a.m. Lobster Dinner and Dance

\section{Thursday, October 1}

10:30 - 11.30 a.m. Coffee at Government House

6:30 - 7.30 p.m. Social Hour

7:30 - 9.00 p.m. Banquet

9:00 - 1.00 p.m. Dance 


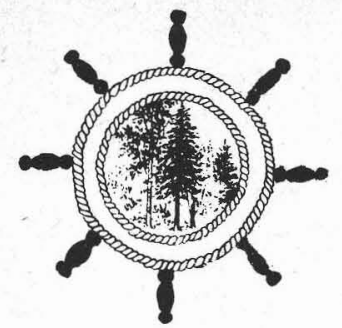

1981 Congrès Annuel

Institut Forestier du Canada

27 septembre - 1 octobre

Hôtel Nova Scotian, Halifax

"Larguons les voiles pour Halifax"

\section{GESTION FORESTIĖRE INTENSIVE}

Samedi, 26 septembre

1:30 - 5.00 p.m. Réunion des Directeurs, I.F.C.

Dimanche, 27 septembre

9:00 - 5.00 p.m. Réunion des Directeurs, I.F.C.

2:00 - 7.00 p.m. Inscription

8:00 - 10.00 p.m. Réception

\section{Lundi, 28 septembre}

$$
\begin{aligned}
\text { 8:00 - } 12.00 \text { midi } & \text { Inscription } \\
\text { 8:00 - } 9.30 \text { a.m. } & \text { Petit déjeuner } \\
\text { 9:30 - } 10.30 \text { a.m. } & \text { Cérémonie d'Ouverture } \\
\text { 10:45 - } 12.00 \text { midi } & \text { Réunion - Partie \#1 } \\
12: 00-2.00 \text { p.m. } & \text { Déjeuner } \\
2: 00-5.00 \text { p.m. } & \text { Conférenciers } \\
& \text { - John Weslien, Chef } \\
& \text { Forestier } \\
& \text { Billeruds AB, la Suède } \\
& \text { - W. Lester Brown, } \\
& \text { Directeur des Opérations } \\
& \text { Forestières, Bowater Inc. } \\
& \text { - Gordon Minnes } \\
& \text { Association Canadienne de } \\
& \text { Pulpe et Papier } \\
& - \text { J. Gordon Ogden III, } \\
& \text { Professeur de Biologie, } \\
& \text { Université Dalhousie }
\end{aligned}
$$

Mardi, 29 septembre

9:00 - 12.00 midi Atelier: Gestion Intensif en Nouvelle Ecosse

12:00 - 2.00 p.m. Déjeuner par la Province de N.E.

2:00 - 5.00 p.m. Atelier: Développements au Canada

Mercredi, 30 septembre

8:30 - 5.00 p.m. Tours (au choix)

\#1 - Récolte et Gestion des Forêts sur les Terres Couronnes et sur les lots privés

\#2 - Gestion des Forêts sur les petits lots

\#3 - Petites industries utilisatrices de bois

6:00 - 1.00 p.m. Dîner aux Homards et Danse

jeudi, 1er octobre

9:00 - 12.00 midi Réunions de petits groupes de travail

12:00 - 2.00 p.m. Déjeuner "Alma Mater"

2:00 - 4.30 p.m. Réunion - Partie \#2

6:30 - 7.30 p.m. Réception

7:30 - 9.00 p.m. Banquet

9:00 - 1.00 a.m. Danse

Vendredi, 2 octobre et samedi, 3 octobre

Possibilité d'une excursion au Cap Breton après la conférence.

\section{PROGRAMME POUR LES CONJOINTES/CONJOINTS}

\section{Dimanche, 27 septembre}

$11: 30$ - 2.30 p.m. Réception pour les Épouses des Directeurs

2:00 - 7.00 p.m. Inscription

8:00 - 10.00 p.m. Réception

\section{Lundi, 28 septembre}

8:00 - 12.00 midi Inscription

8:00 - 9.30 a.m. Petit déjeuner

10:30 - 5.30 p.m. Tour de la vallée Annapolis

\section{Mardi, 29 septembre}

10:00 - 11.30 a.m. Promenade Historique

12:00 - 2.00 p.m. Déjeuner par la Province de N.E.

2:30 - 3.30 p.m. Parade de Mode
Mercredi, 30 septembre

10:00 - 12.00 midi Petites excursions en ville et aux musées

12:00 - 2.00 p.m. Déjeuner

2:00 - 4.00 p.m. Tour en bateau

6:00 - 1.00 a.m. Dîner aux Homards et Danse

Jeudi, 1er octobre

10:30 - 11.30 a.m. Café à l'Hôtel du Gouvernement

6:30 - 7.30 p.m. Réception

7:30 - 9.00 p.m. Banquet

9:00 - 1.00 a.m. Danse 